Kulka, D., Preston, J. M. \& Walker, T. K. (1951). J. gen. Microbiol. 5, 18-21.

\title{
The Photographic Examination of Giant Colonies
}

\author{
By DORA KULKA, J. M. PRESTON AND T. K. WALKER \\ College of Technology, University of Manchester
}

\begin{abstract}
SUMMARY: An apparatus was designed to photograph colonies of micro-organisms on Petri dishes either under conditions of dark-field illumination or of top-lighting. It serves to reveal any motility of the organisms and is valuable as an aid to identification, particularly in the genus Acetobacter, members of which have characteristic giant colonies.
\end{abstract}

Recently (1949) we recorded particulars of giant colonies of Acetobacter species which had been grown on a semi-solid medium. In such conditions certain species, e.g., $A$. aceti (Kützing) Beijerinck, form highly characteristic colonies. Other species, including the majority of the known species of Acetobacter, characterized by colonies of a particular type, can be classified in a number of groups. Species which form cellulose, e.g. A. xylinum (Brown) Holland, $A$. kützingianum (Hansen) Bergey et al., A acetigenum (Henneberg) Bergey et al. belong to one group. Again, the giant colonies of $\boldsymbol{A}$. acetosum (Henneberg) Bergey et al., $\boldsymbol{A}$. ascendens (Henneberg) Bergey et al., and $A$. rancens Beijerinck, though differing from each other in size, are similar in general appearance and form another group. A third group comprises $A$. suboxydans Kluyver \& de Leeuw, and a number of species all of which are highly motile and form colonies with zones of motile cells. These various characteristic features are brought out particularly well in photographs taken under conditions which we have devised, and since the technique may prove of service to others, we describe it below.

\section{CONDITIONS FOR OBTAINING PHOTOGRAPHS OF PETRI-DISH CULTURES OF MICRO-ORGANISMS}

The visibility of small objects depends on their power to reflect, to refract and to scatter light. The smaller the objects the more important is the scattering effect and the less important is reflexion or refraction. The optimum conditions for observing scattering from small objects can be deduced from Rayleigh's equation (1899). Zimm, Stein \& Doty (1945), in a recent review, give an expression for the scattering of unpolarized light by objects much smaller than a wavelength. For the present purpose this can be set out as:

$$
I \propto\left(1+\cos ^{2} \theta\right),
$$

where $I$ is the intensity of the scattered light and $\theta$ is the angle between the incident beam and the direction of scattering. From this expression it follows that the maxima will be found in the scattered light when $\theta=0$ and $180^{\circ}$, i.e. when the scattering is backwards or forwards in the direction of incidence. With objects approaching one wavelength of light in size, such as many microorganisms, there is appreciable interference between the scattered rays in the backward direction, which eliminates the backward maximum. There is then 
only one maximum in the scattered light, that in the forward direction, which is therefore the best direction in which to look for scattered light indicative of the presence of microscopic objects. In the precise direction of incidence there is much unscattered light from the direct beam which will outshine the scattered light, so the path of the direct beam must be avoided, and the best direction in which to look will be just to one side of the direct beam.

Reflexion and refraction can be considered together, because they are both aspects of the phenomena resulting from the difference of the refractive indices of the object and its environment. When this difference is small, as it is with micro-organisms suspended in solid media, it can be shown that the greatest intensity of both refraction and reflexion from random objects lies near to the path of the direct beam. This direction is therefore the best in which to look for reflected and refracted light indicative of the presence of microscopic objects.

\section{METHOD OF EXAMINATION}

The above analysis indicates that the micro-organisms under examination should be illuminated in such a way that the eye of the observer (or the observation lens) will just, but only just, escape the direct beam used for illuminating the preparation. These conditions are therefore optimum for maximum visibility if used with a good black background against which the faintly scattered light is most clearly perceived. To get even illumination for photography the light must come from all sides. This condition is best fulfilled with an annular cone of rays symmetrically disposed about the optic axis of the camera. The required conditions were obtained with a simple apparatus shown in Pl. 1, fig. 1, and diagrammatically in Fig. 1. As shown in the diagram (Fig. 1) the apparatus consists of an annular frame $A$ which bears a ring of nine 12 V. $24 \mathrm{~W}$. motor-car headlamp bulbs $O$, connected in series and run direct from the $110 \mathrm{~V}$. mains. (Bulbs $(24 \mathrm{~V}$.) could be run from 220 to $230 \mathrm{~V}$. mains, or alternatively a transformer could be used to step down the mains-voltage and the lamps could be run in parallel.) A central stop, which provides a black background is obtained with a cylinder $\boldsymbol{B}$ of black paper, closed at the bottom. The incident light is further limited by a diaphragm of black paper $C$. Stouter forms of $B$ and $C$ can be made from tin. The Petri dish containing the culture $D$ to be examined is placed on a ring $E$ which is clamped to the support which also bears the ring of lamps and the camera. The support is shown in Fig. 1, where the path of the light rays $O L$ is indicated. The central stop $B$ and the diaphragm $C$ prevent any direct rays reaching the camera lens. It will be seen that the light has only to be deviated through a small angle to reach the camera lens. Thus the illuminating and viewing directions are arranged in the best way to detect faint, scattered, reflected and refracted light from the culture.

To show the raised growth of certain of the cultures the lighting was varied from that in Fig. 1. For deviations from plane surfaces, top-lighting is best, but should not be inclined at too great an angle to the surface, or the elevations and depressions become imperceptible. Top-lighting is obtained by raising the ring of lamps above the plane of the Petri dish. To emphasize the elevation of the 
colonies still further the light from half the ring of lamps is shaded with black paper and the light from the unshaded lamps is slightly diffused with a semicircular strip of matt celluloid. The photographs in Pl. 1, figs. 2 and 3, and Pl. 2, figs. 4-7, were taken with this illumination.

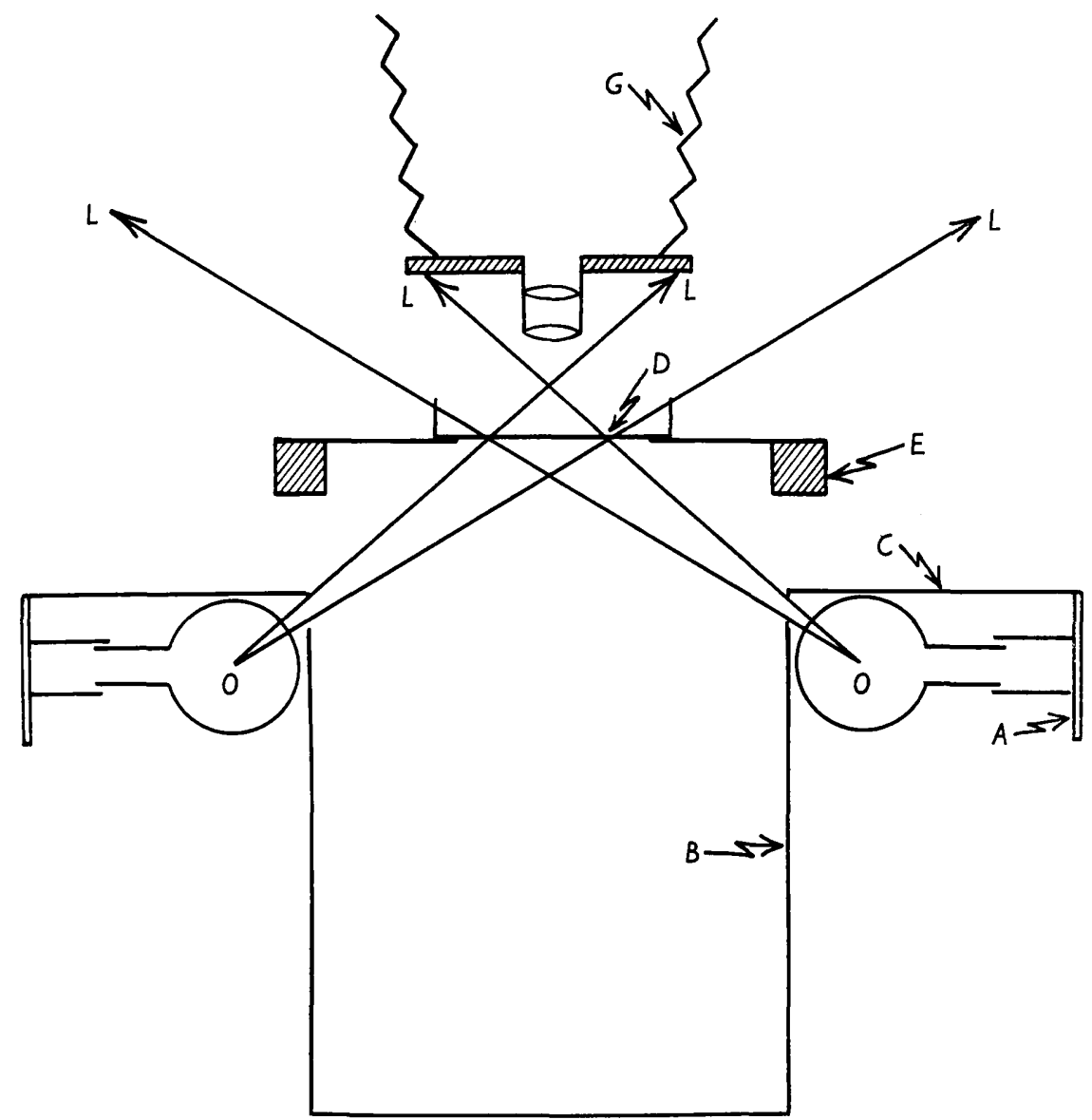

Fig. 1. Diagrammatic cross-section of the apparatus.

\section{RESULTS}

Pl. 1, figs. 2 and 3, show a colony of $A$. acetosum photographed with bottomand top-lighting, respectively. The colony of $A$. acetigenum shown in Pl. 2, figs. 4 and 5, was similarly photographed. Pl. 2, fig. 6, shows a colony of A. kützingianum, a species which, in certain media, forms a cellulose surface pellicle. The strong resemblance of this giant colony to that of $A$. acetigenum, which is also a cellulose-producing organism, may be noted. $A$. xylinum, another cellulose former, we found gave giant colonies similar to, though smaller than, those of $A$. kützingianum and $A$. acetigenum. Finally, Pl. 2, fig. 7 , shows the large zone of motile cells to be observed in giant colonies of A. suboxydans. The strain of $A$. suboxydans used in this work was N.C.T.C. 
Journal of General Microbiology, Vol. 5, No. 1

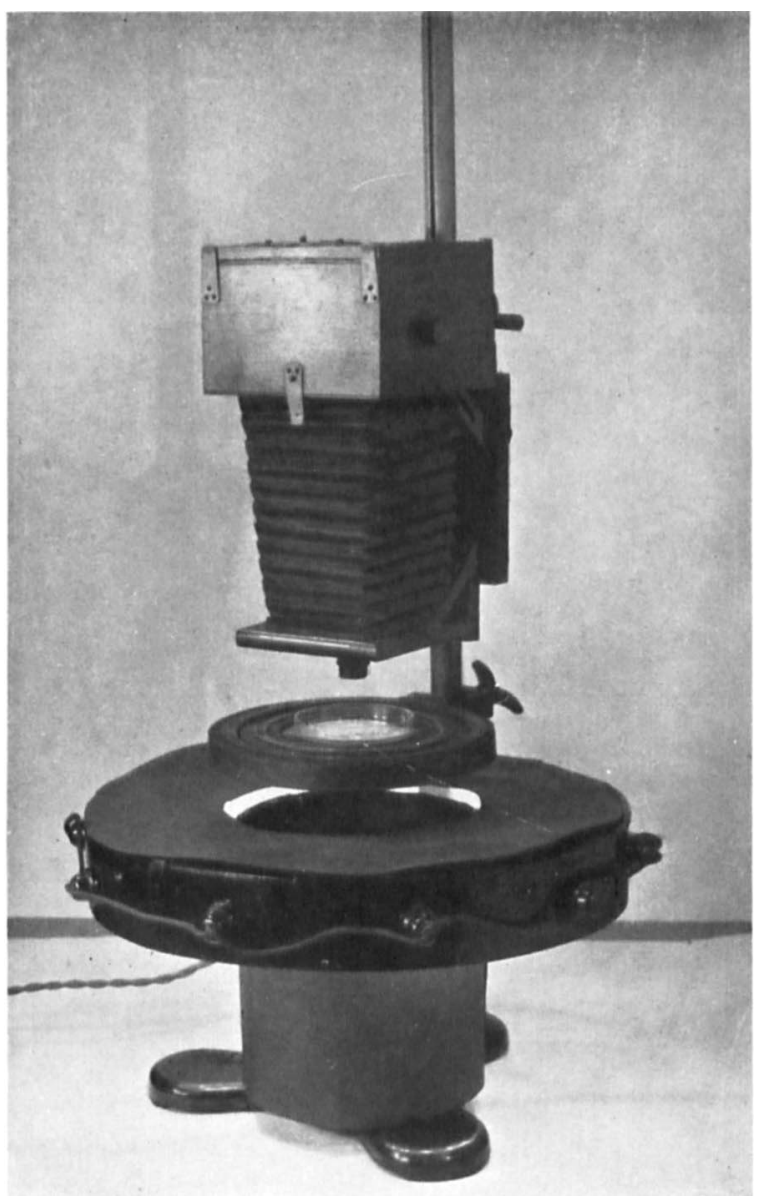

Fig. 1

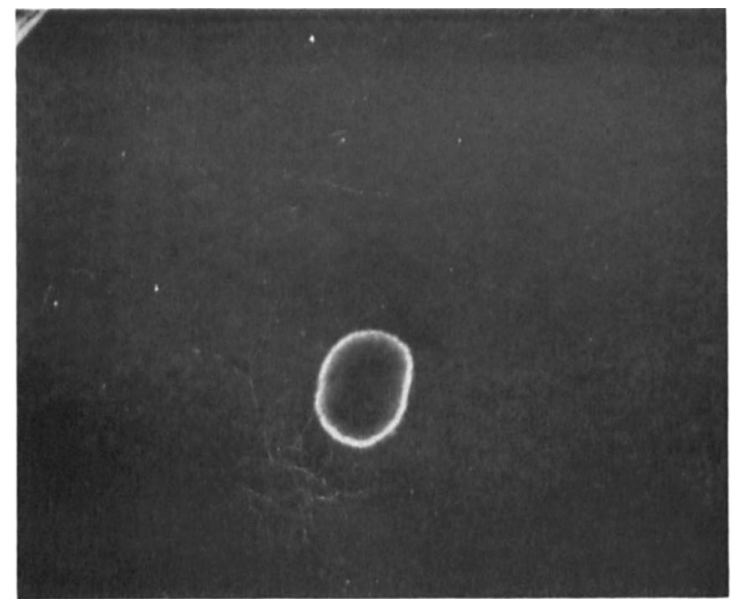

Fig. 2

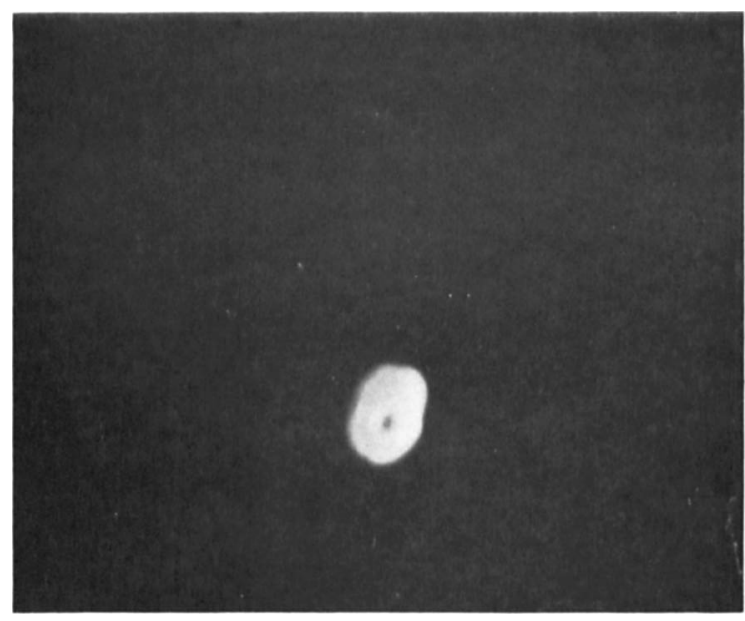

Fig. 3

D. Kulka, J. M. Preston and T. K. Walker-Photograpity of giant colonies. Pidate 1 
Journal of General Microbiology, Vol. 5, No. 1

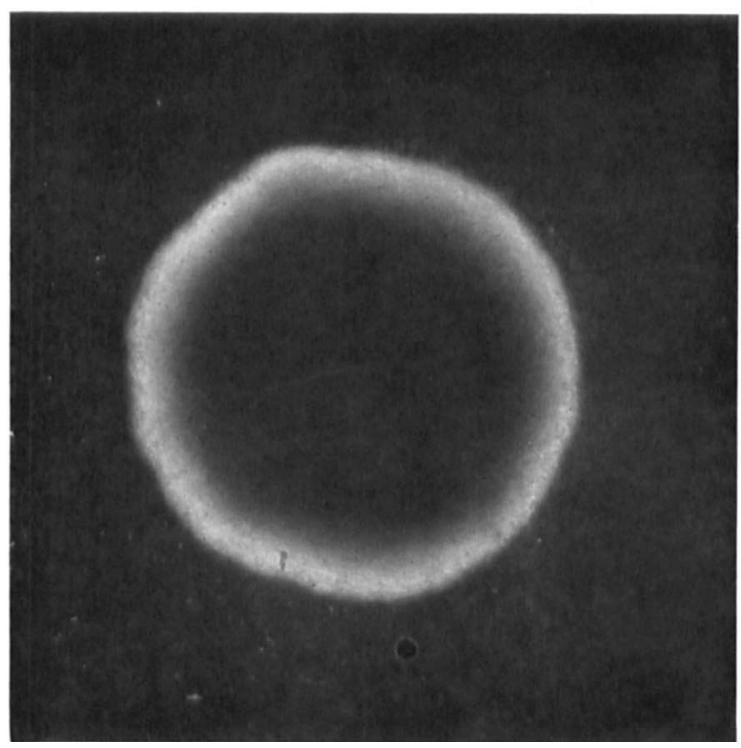

Fig. 4

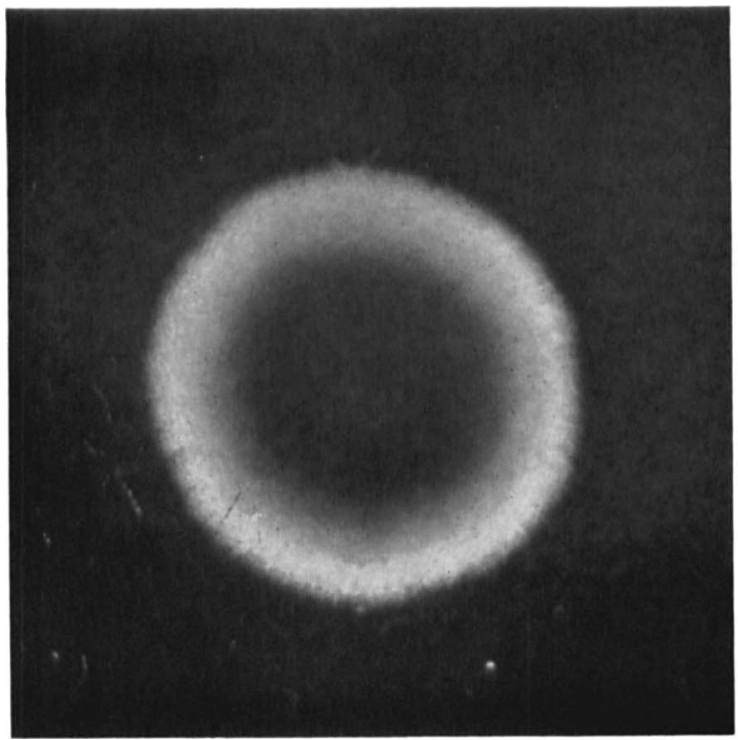

Fig. 6

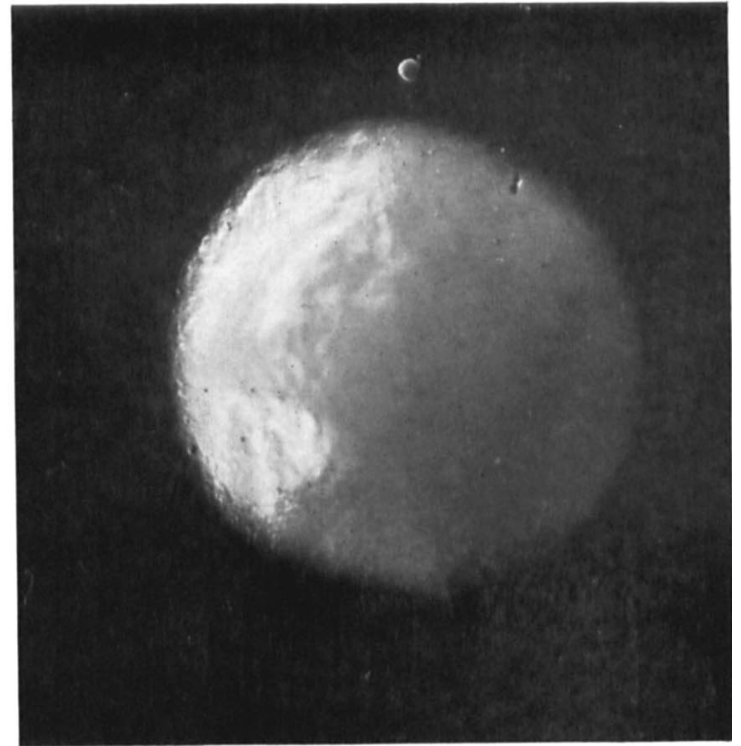

Fig. 5

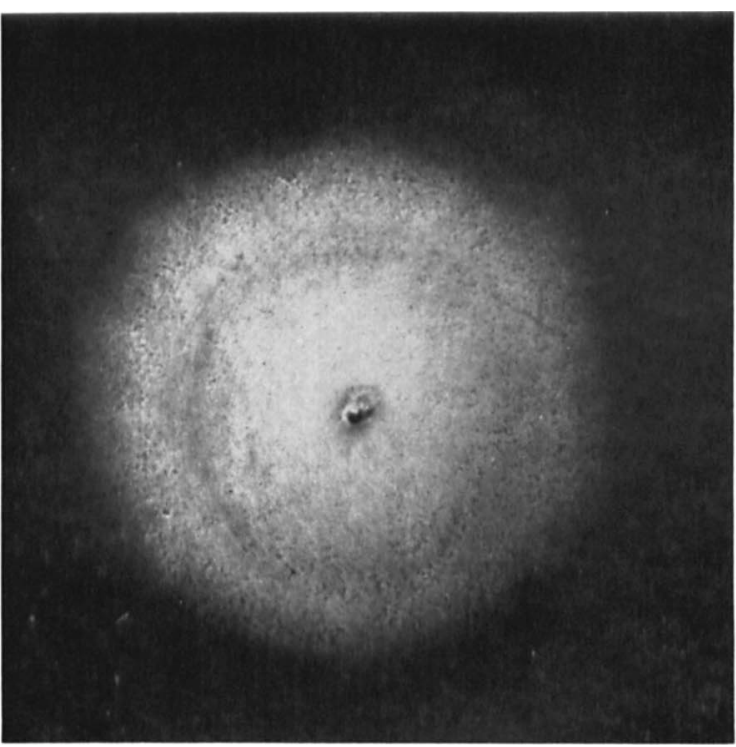

Fig. 7

D. Kulka, J. M. Preston and T. K. Walker-Photography of giant colonies. Plate 2 
7069 (A.T.C.C. 621) and was obtained, as were also the other species, from the National Collection of Type Cultures. Other strains of $A$. suboxydans produced giant colonies strongly resembling that shown in $\mathrm{Pl}$. 2, fig. 7 .

\section{REFERENCES}

RAYleigh, LoRd (1899). On the transmission of light through an atmosphere containing small particles in suspension and on the origin of the blue of the sky. Phil. Mag. 47, 377.

Kulka, D., Preston, J. M. \& Walker, T. K. (1949). Giant colonies of Acetobacter species as an aid to identification. J. Inst. Brew. 55, 141.

Zimm, B. H., Stein, R. S. \& Doty, P. (1945). Classical theory of light scattering from solutions-a review. Polymer Bull. 1, nos. 4/5, 90.

\section{EXPLANATION OF PLATES}

Fig. 1. General view of the apparatus.

\section{Plate 1}

Fig. 2. One colony of $A$. acetosum N.C.T.C. 2224. Dark-field illumination. $\times \mathbf{2 \cdot 9}$.

Fig. 3. Same colony of A. acetosum as in fig. 2. Top-lighting. $\times \mathbf{2 \cdot 9}$.

\section{Plate 2}

Fig. 4. A. acetigenum N.C.T.C. 5346. Dark-field illumination. $\times \mathbf{2 . 9}$.

Fig. 5. A. acetigenum N.C.T.C. 5346. Same colony as fig. 4 . Top-lighting. $\times \mathbf{2 \cdot 9}$.

Fig. 6. A. kützingianum N.C.T.C. 3924. Dark-field illumination. $\times \mathbf{2 \cdot 9}$.

Fig. 7. A. suboxydans N.C.T.C. 7069 (A.T.C.C. 621). Dark-field illumination. $\times 2.9$.

(Received 3 November 1949) 